\title{
Distinguishability of non-orthogonal density matrices does not imply violations of the second law*
}

\author{
Piero G. Luca Mana ${ }^{\dagger}$ \\ Laboratory of Quantum Electronics and Quantum Optics (QEO), \\ Department of Microelectronics and Information Technology (IMIT), \\ Royal Institute of Technology (KTH), Isafjordsgatan 22, SE-16440 Stockholm, Sweden
}

(Dated: 30 January 2005)

\begin{abstract}
The hypothetical possibility of distinguishing preparations described by non-orthogonal density matrices does not necessarily imply a violation of the second law of thermodynamics, as was instead stated by von Neumann. On the other hand, such a possibility would surely mean that the particular density-matrix space (and related Hilbert space) adopted would not be adequate to describe the hypothetical new experimental facts. These points are shown by making clear the distinction between physical preparations and the density matrices which represent them, and then comparing a "quantum" thermodynamic analysis given by Peres with a "classical" one given by Jaynes.
\end{abstract}

PACS numbers: 03.65.Ca,65.40.Gr,03.67.-a

\section{VON NEUMANN (AND PERES) ON ORTHOGONALITY AND THE SECOND LAW}

In $\S$ V. 2 of von Neumann's Mathematische Grundlagen der Quantenmechanik [1] we find the following two propositions (p. 197): ${ }^{1}$

two states $\phi, \psi[\ldots]$ can certainly be separated by a semi-permeable membrane if they are orthogonal;

and the converse

if $\phi, \psi$ are not orthogonal, then the assumption of such a semi-permeable membrane contradicts the second law [of thermodynamics].

The demonstrations of these two statements given by von Neumann are based on the same "thermodynamic considerations" by which he derives his entropy formula. Peres, in his insightful and lucid book [2], also gave a demonstration to show that if we were able to produce two semi-permeable membranes which unambiguously distinguish non-orthogonal "states", we could violate the second law of thermodynamics.

The purpose of this paper is first to rephrase the two statements above, substituting the ambiguous term "state" with other terms which make clear the distinction between a physical phenomenon and its mathematical description [2-13], and then to show that von Neumann's (and Peres') second statement, once rephrased, is not necessarily true. This will be done by comparing Peres' demonstration [2] with a lucid, simple, and probably less known analysis, given by Jaynes [14], of a seeming violation of the second law of thermodynamics apparently due to classical (i.e. non-quantum) "indistinguishability" issues.

\footnotetext{
*Dedicated to the memory of Asher Peres

${ }^{\dagger}$ Electronic address: mana@imit.kth. se

1 “zwei Zustände $\phi, \psi[\ldots]$ durch semipermeable Wände bestimmt getrennt werden können, wenn sie orthogonal sind", and "sind $\phi, \psi$ nicht orthogonal, so widerspricht die Annahme einer solchen semipermeablen Wand dem zweiten Hauptsatz".
}

The paper is not directly concerned with questions about the relation between thermodynamics and statistical mechanics, nor to questions about "classical" or "quantum" entropy formulae. Indeed, no particular entropy formula will be used, but only the assumption that in a closed thermodynamic cycle the entropy change vanishes.

\section{EXEGESIS OF VON NEUMANN'S STATEMENTS}

Let me now rephrase von Neumann's statements above, at the cost of making some semantic violence to them, and in particular let me eliminate the too-many-faced term "state" in favour of the two distinct terms (physical) preparation [2-13] (cf. also [15-17]), which has a clear physical, experimental meaning, and statistical matrix, which denotes a mathematical object instead: 'statistical matrix' is the old term for what has been called 'density matrix' apparently since Wigner [18] (cf. Fano [19]); since the matrices we consider here concern statistics but no probability densities, I prefer to use the old term hereafter.

Let me also substitute, but only for the time being, the thermodynamic concept of a semi-permeable membrane with the more general idea of an observation procedure [2-11]. Von Neumann spoke of membranes only because he needed a conceptual device that could endow with thermodynamic consequences the ability to distinguish (and thus separate) physical preparations. We shall reintroduce and discuss this device in the next section, but for the moment let us be more general.

We can express von Neumann's first statement (1) as follows:

if there is an observation procedure by which we can distinguish two physical preparations with certainty, then we mathematically represent the latter by orthogonal statistical matrices; and vice versa, by non-orthogonal statistical matrices if we do not know of any such observation procedure.

(Two statistical matrices $\phi$ and $\psi$ are said to be orthogonal if and only if $\operatorname{tr}(\phi \psi)=0$.) This re-statement makes clear the 
difference between the physical phenomenon and the mathematical objects used to describe, or represent, it: we see that von Neumann's original proposition, which looked mathematical in nature, disguises in fact a statement about physics methodology. Note also that from a logical point of view ${ }^{2}$ I have completed von Neumann's original proposition

$$
\text { orthogonality } \Rightarrow \text { distinguishability }
$$

into the two

$$
\begin{aligned}
\text { distinguishability } & \Rightarrow \text { orthogonality, } \\
\text { indistinguishability } & \Rightarrow \text { non-orthogonality, }
\end{aligned}
$$

or equivalently

$$
\text { distinguishability } \Leftrightarrow \text { orthogonality. }
$$

(The reader should pay attention not to confuse the "causal" connexion, in a loose sense, with the logical connexion which exist between distinguishability and orthogonality. The causal connexion goes only in one direction: a physicist uses orthogonal matrices because the corresponding preparations are distinguishable, but two preparations do not become suddenly distinguishable just because she has represented them on paper by two orthogonal matrices. The logical connexion goes instead both ways: if a physicist can distinguish two preparations, we can deduce that she will represent them by orthogonal matrices; and if we see that a physicist represents two preparations by orthogonal matrices, we can deduce that she knows of an observation procedure that can distinguish those preparations. The present author confused these two kind of connexions himself in the first drafts of this paper. This kind of confusion between physics and probability is called "the mind-projection fallacy" by Jaynes [21-23].)

With regard to von Neumann's (and Peres') second proposition (2), it can be rephrased as follows:

if we could distinguish, by means of some observation procedure, two physical preparations represented by non-orthogonal statistical matrices, then we could violate the second law of thermodynamics.

This proposition makes as well a clear distinction between physical phenomenon and mathematical description; however, it is logically intrinsically vain. Let us see why from a logical point of view first. The antecedent of the proposition $^{3}$ formally is 'distinguishability $\wedge$ non-orthogonality', but this is false in view of the previous statement (1'), namely

\footnotetext{
2 In the following I use the two logical symbols ' $\Rightarrow$ ' ('implies', 'if ... then') for logical implication, and ' $\wedge$ ' ('and') for logical conjunction [20].

${ }^{3}$ In a proposition of the form ' $A \Rightarrow B$ ', $A$ is called the antecedent (or implicans, or protasis) and $B$ the consequent (or implicate, or apodosis) [20].
}

'distinguishability $\Rightarrow$ orthogonality': remember that if ' $A \Rightarrow$ $B$ ' holds, then we cannot have both $A$ true and $B$ false [20]. But we know that from a false antecedent one can idly deduce any proposition whatever [20], hence the statements (2) and (2') are devoid of real content. Speaking on a less abstractly logical level, the point is that if we can distinguish two preparations represented by non-orthogonal statistical matrices, then we are evidently no longer following our prescription (1') to mathematically represent preparation distinguishability by means of matrix orthogonality; we are inconsistent. Any consequences we derive, like e.g. violations of the second law, are likely to be only artifacts of our inconsistency rather than real phenomena. We must thus amend the particular statistical matrices or the whole statistical-matrix space used, since they have not been adequately chosen to describe the physical phenomenon in question or our experimental capabilities.

I shall in fact show explicitly that the violation of the second law of statements (2) and (2') is only an artifact, by analysing Peres' demonstration [2] and comparing it with Jaynes' already mentioned analysis [14] of an analogous seeming violation of the second law of thermodynamics due to entirely classical "indistinguishability" issues. ${ }^{4}$ In order to do this, let us analyse the way in which von Neumann and Peres link quantum distinguishability and non-orthogonality with the second law of thermodynamics.

\section{3. “QUANTUM” IDEAL GASES, SEMI-PERMEABLE MEMBRANES, AND THERMODYNAMICS}

In order to analyse a proposition which relates distinguishability of quantum preparations with the second law of thermodynamics, it is necessary to introduce a thermodynamic body possessing "quantum" characteristics, i.e., quantum degrees of freedom.

Let us first recall that ("classical") ideal gases are defined as homogeneous, uniform thermodynamic bodies characterisable by two thermodynamic variables: the volume $V>0$ and the temperature $T>0$, and for which the internal energy is a function of the variable $T$ alone, ${ }^{5}$ this implies, via the first law of thermodynamics, that in any isothermal process the work done by the gas, $W$, is always equal to the heat absorbed by the gas, $Q$ :

$$
\left.W=n R T \ln \left(V_{\mathrm{f}} / V_{\mathrm{i}}\right)=Q \quad \text { (isothermal processes }\right)
$$

\footnotetext{
4 Jaynes used his analysis to show that the definition and the quantification of the thermodynamic entropy depend on the particular thermodynamic variables that define the thermodynamic system: a long known fact, which also Grad [24-26], who had different views on statistical mechanics than Jaynes', stressed.

${ }^{5}$ See e.g. the very fine little book by Truesdell and Bharatha [27].
} 
where $V_{\mathrm{i}}$ and $V_{\mathrm{f}}$ are the volumes at the beginning and end of the process, $R$ is the molar gas constant, and $n$ is the (constant) number of moles. This formula will be true throughout the paper, as we shall only consider isothermal processes.

One often considers samples of such ideal gases in a chamber and inserts, moves, or removes impermeable or semipermeable membranes ${ }^{6}$ at any position one pleases in order to subject the samples, independently of each other, to variations of e.g. volume or pressure. ${ }^{7}$

We must now face the question of how to introduce and mathematically represent quantum degrees of freedom in an ideal gas. Von Neumann [1, §V.2] used a hybrid classicalquantum description, microscopically modelling a "quantum" ideal gas as a quantity $n$ of classical particles (for simplicity) possessing an "internal" quantum degree of freedom represented by a statistical matrix $\rho$ living in an appropriate statistical-matrix space; this space and the statistical matrix are always assumed to be the same for all the gas particles. He then treated two gas samples described by different statistical matrices as gases of somehow different chemical species. ${ }^{8}$ This conceptual device presents some problems, to be discussed more in detail elsewhere [38]; for example, the chemical species of a gas is not a thermodynamic variable, and even less a continuous one: chemical differences cannot change continuously to zero. ${ }^{9}$ Intuitively, it would seem more appropriate to somehow describe a quantum ideal gas by the variables $(V, T, \boldsymbol{\rho})$ instead, taking values on appropriate sets.

However, we shall follow von Neumann and Peres instead and speak of a ' $\phi$-gas', or a ' $\psi$-gas', etc., where $\phi$ or $\psi$ are the statistical matrices describing its quantum degrees of freedom, just as if we were speaking of gases of different chemical species (like e.g. 'argon' and 'helium'). ${ }^{10}$ The thermodynamic variables are only $(V, T)$ for each such gas.

\footnotetext{
${ }^{6}$ Partington $[28, \S 28]$ informs us that these were first used in thermodynamics by Gibbs [29].

${ }^{7}$ Although the conclusions drawn from such kind of reasonings are often valid, it must be said that the mathematical description adopted is wanting (Truesdell often denounced the fact that classical thermodynamics has only very rarely been treated with the conceptual respect and mathematical dignity which are accorded to other theories like rational mechanics or general relativity). The correct formalism to describe insertions and removals of membranes should involve field quantities (cf. Buchdahl [30, §§46, 75] and see e.g. Truesdell [31, lectures 5, 6 and related appendices] or references [32-36]), as indicated by the possibility of introducing as many membranes as we wish and hence to control smaller and smaller portions of the gas.

8 The idea had been presented by Einstein eighteen years earlier [37], but it is important to point out that for Einstein the "internal quantum degree of freedom" was just a "resonator" capable of assuming only discrete energies (i.e., it was not described by statistical matrices, and non-orthogonality issues were unknown); thus his application was less open to problems and critique than von Neumann's.

${ }^{9}$ An observation made by Partington [28, §II.28] in reference to Larmor [39, p. 275].

${ }^{10}$ Dieks and van Dijk [40] point rightly out that for a $\boldsymbol{\phi}$-gas one should more correctly consider the total statistical matrix $\bigotimes_{i=1}^{N} \phi$, where $N=n N_{\mathrm{A}}$ (with $N_{\mathrm{A}}$ Avogadro's constant) is the total number of particles.
}

Two samples of these quantum ideal gases can be (more or less effectively) separated by semi-permeable membranes, analogous to those used with chemically different gases. The microscopic idea [1, p. 196][2, p. 271] is, paraphrasing von Neumann, to construct many windows in the membrane, each of which is made as follows: each particle of the gases is detained there and an observation is performed on its quantum degrees of freedom. Depending on the observation result, the particle penetrates the window or is reflected, with unchanged momentum. This implies that the number of particles and hence the pressures or volumes of the gases on the two sides of the semi-permeable membrane will vary, and may set the membrane in motion, producing work (e.g. by lifting a weight which loads the membrane). In more general terms, this membrane is a device which performs a physical operation, described by a given positive-operator-valued measure (POVM) and completely positive map (CPM) [41-44], on the quantum degrees of freedom of the gases' particles, and depending on the result it acts on their translational degrees of freedom, e.g. separating them spatially. There arises thus a kind of mutual dependence between the quantum degrees of freedom and the thermodynamic parameters (like the volume $V$ ) of a quantum ideal gas.

Let us make an example. Imagine a chamber having volume $V$ and containing a mixture of a quantity $n / 2$ of a $z^{+}$-gas and $n / 2$ of a $z^{-}$-gas, i.e., of two quantum ideal gases whose quantum degrees of freedom are represented by the statistical matrices

$$
\begin{aligned}
& z^{+} \stackrel{\text { def }}{=}\left|z^{+}\right\rangle\left\langle z^{+}\right| \hat{=}\left(\begin{array}{ll}
1 & 0 \\
0 & 0
\end{array}\right), \\
& z^{-} \stackrel{\text { def }}{=}\left|z^{-}\right\rangle\left\langle z^{-}\right| \hat{=}\left(\begin{array}{ll}
0 & 0 \\
0 & 1
\end{array}\right),
\end{aligned}
$$

in the usual spin-1/2 notation (Fig. 1, step a). These two statistical matrices represent quantum preparations that can be distinguished with certainty, hence there exists a semi-permeable membrane, of the kind described above, which is completely opaque to (the particles of) the $z^{-}$-gas and completely transparent to (the particles of) the $z^{+}$-gas. Analogously, there exists another semi-permeable membrane with the opposite properties, viz. it is completely opaque to (the particles of) the $z^{+}$-gas and completely transparent to (the particles of) the $z^{-}$gas. Such membranes implement the two-element POVM $\left\{z^{ \pm}\right\}$ with outcomes given by the CPMs $\left\{\rho \mapsto z^{ \pm} \rho z^{ \pm} / \operatorname{tr}\left(z^{ \pm} \rho z^{ \pm}\right)\right\}$: acting on the $z^{+}$-gas it yields ${ }^{11}$

$$
\begin{aligned}
z^{+} \mapsto z^{+} & \text {with probability } \operatorname{tr}\left(z^{+} z^{+} z^{+}\right)=1, \\
z^{+} \mapsto z^{-} & \text {with probability } \operatorname{tr}\left(z^{-} z^{+} z^{-}\right)=0,
\end{aligned}
$$

\footnotetext{
${ }^{11}$ Due to the large number of gas particles considered, the outcome probabilities are numerically equal, within small fluctuations negligible in the present work, to the average fraction of gas correspondingly transmitted or reflected by the membranes.
} 


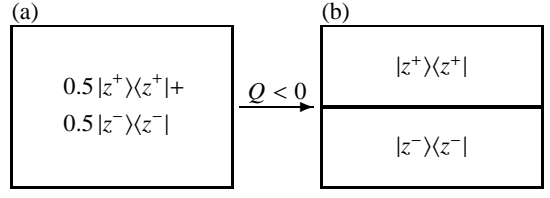

Figure 1: Separation of completely distinguishable quantum gases

while acting on the $z^{-}$-gas it yields

$$
\begin{array}{ll}
z^{-} \mapsto z^{+} & \text {with probability } \operatorname{tr}\left(z^{+} z^{-} z^{+}\right)=0, \\
z^{-} \mapsto z^{-} & \text {with probability } \operatorname{tr}\left(z^{-} z^{-} z^{-}\right)=1
\end{array}
$$

(note that these are just von Neumann projections), i.e., the operation separates the two preparations which certainty. The only difference between the membranes is in which kind of gas (particle) they let through.

Now, imagine to insert these two membranes in the chamber, very near to its top and bottom walls respectively. Pushing them isothermally toward the middle of the chamber, they will separate the two gases and in the end we shall have the $z^{+}$-gas completely above the first membrane and the $z^{-}$-gas completely under the other in contact with the first (step b). In order to move these membranes and achieve this separation we have to spend an amount of work equal to

$$
-2 \times \frac{n}{2} R T \ln \frac{V / 2}{V} \approx 0.693 n R T
$$

because each membrane must overcome the pressure exerted by the gas to which it is opaque. Since the process is isothermal, the quantity above is also the (positive) amount of heat released by the gases.

The semi-permeable membranes can also be used to realise the inverse process, i.e. the mixing of two initially separated $z^{+}$- and $z^{-}$-gases. In this case Eq. (7) would be the amount of heat absorbed by the gases as well as the amount of work performed by them.

However, the quantum degrees of freedom of two gases may also be prepared in such a way that no observation procedure can distinguish between them with certainty, and hence there is no semi-permeable membrane which can separate them completely; this has of course consequences for the amount of work that can be gained by using the membrane. Let us make another example. Imagine again the initial situation above, but this time with a mixture of a quantity $n / 2$ of a $\boldsymbol{z}^{+}$-gas and $n / 2$ of an $\boldsymbol{x}^{+}$-gas, with

$$
\boldsymbol{x}^{+} \stackrel{\text { def }}{=}\left|x^{+}\right\rangle\left\langle x^{+}\right| \hat{=} \frac{1}{2}\left(\begin{array}{ll}
1 & 1 \\
1 & 1
\end{array}\right) \text {. }
$$

The two statistical matrices $z^{+}$and $\boldsymbol{x}^{+}$are non-orthogonal, $\operatorname{tr}\left(z^{+} x^{+}\right) \neq 0$, and correspond to quantum preparations that cannot be distinguished with certainty, and so there do not exist semi-permeable membranes which are completely opaque to (the particles of) the one gas and completely transparent to (the particles of) the other gas. Mathematically this is reflected in the non-existence of some two-outcome POVM $\left\{\boldsymbol{A}_{1}, \boldsymbol{A}_{2}\right\}$ with the properties

$z^{+}$yields first outcome with probability $\operatorname{tr}\left(\boldsymbol{A}_{1} z^{+} \boldsymbol{A}_{1}\right)=1$,

$z^{+}$yields second outcome with probability $\operatorname{tr}\left(\boldsymbol{A}_{2} z^{+} \boldsymbol{A}_{2}\right)=0$,

and

$\boldsymbol{x}^{+}$yields first outcome with probability $\operatorname{tr}\left(\boldsymbol{A}_{1} \boldsymbol{x}^{+} \boldsymbol{A}_{1}\right)=0$,

$\boldsymbol{x}^{+}$yields second outcome with probability $\operatorname{tr}\left(\boldsymbol{A}_{2} \boldsymbol{x}^{+} \boldsymbol{A}_{2}\right)=1$.

It can be shown $[1,2]$ that in this case the operation which will require the maximum amount of work is represented by the two-element POVM $\left\{\boldsymbol{\alpha}^{ \pm}\right\}$, where

$$
\begin{aligned}
\alpha^{ \pm} & \stackrel{\text { def }}{=}\left|\alpha^{ \pm}\right\rangle\left\langle\alpha^{ \pm}\right| \hat{=} \frac{1}{4}\left(\begin{array}{cc}
2 \pm \sqrt{2} & \pm \sqrt{2} \\
\pm \sqrt{2} & 2 \mp \sqrt{2}
\end{array}\right), \\
\left|\alpha^{ \pm}\right\rangle & \stackrel{\text { def }}{=}(2 \pm \sqrt{2})^{-\frac{1}{2}}\left(\left|z^{ \pm}\right\rangle \pm\left|x^{ \pm}\right\rangle\right), \\
& \equiv \frac{1}{2}\left[ \pm(2 \pm \sqrt{2})^{\frac{1}{2}}\left|z^{+}\right\rangle+(2 \mp \sqrt{2})^{\frac{1}{2}}\left|z^{-}\right\rangle\right],
\end{aligned}
$$

whose outcomes are given by the CPMs (projections) $\rho \mapsto$ $\boldsymbol{\alpha}^{ \pm} \boldsymbol{\rho} \boldsymbol{\alpha}^{ \pm} / \operatorname{tr}\left(\boldsymbol{\alpha}^{ \pm} \boldsymbol{\rho} \boldsymbol{\alpha}^{ \pm}\right)$. Note that the Hilbert-space vectors $\left|\alpha^{ \pm}\right\rangle$ are the eigenvectors of the matrix $\lambda$, where

$$
\lambda \stackrel{\text { def }}{=} \frac{1}{2} z^{+}+\frac{1}{2} x^{+}=\frac{2+\sqrt{2}}{4} \alpha^{+}+\frac{2-\sqrt{2}}{4} \alpha^{-} \hat{=} \frac{1}{4}\left(\begin{array}{ll}
3 & 1 \\
1 & 1
\end{array}\right),
$$

and they are orthogonal, so that $\operatorname{tr}\left(\boldsymbol{\alpha}^{+} \boldsymbol{\alpha}^{-}\right)=0$. The action of this POVM on the statistical matrices of our gases is given by

$$
\begin{aligned}
z^{+} \mapsto \alpha^{+} \quad \text { with probability } \operatorname{tr}\left(\boldsymbol{\alpha}^{+} z^{+} \boldsymbol{\alpha}^{+}\right) & =(2+\sqrt{2}) / 4 \\
& \approx 0.854, \\
z^{+} \mapsto \boldsymbol{\alpha}^{-} \quad \text { with probability } \operatorname{tr}\left(\boldsymbol{\alpha}^{-} z^{+} \boldsymbol{\alpha}^{-}\right) & =(2-\sqrt{2}) / 4 \\
& \approx 0.146,
\end{aligned}
$$

and

$$
\begin{array}{ll}
\boldsymbol{x}^{+} \mapsto \boldsymbol{\alpha}^{+} & \text {with probability } \operatorname{tr}\left(\boldsymbol{\alpha}^{+} \boldsymbol{x}^{+} \boldsymbol{\alpha}^{+}\right) \approx 0.854, \\
\boldsymbol{x}^{+} \mapsto \boldsymbol{\alpha}^{-} & \text {with probability } \operatorname{tr}\left(\boldsymbol{\alpha}^{-} \boldsymbol{x}^{+} \boldsymbol{\alpha}^{-}\right) \approx 0.146
\end{array}
$$

The significance of the equations above is that the half/half mixture of $z^{+}$- and $\boldsymbol{x}^{+}$-gases can equivalently be treated as a $0.854 / 0.146$ mixture of an $\boldsymbol{\alpha}^{+}$-gas and an $\boldsymbol{\alpha}^{-}$-gas, as is now shown.

We can construct two semi-permeable membranes which implement the above POVM and CPMs in such a way that they can be used to completely separate an $\alpha^{+}$-gas from an $\alpha^{-}$-gas, i.e., one membrane is totally transparent to the former and totally opaque to the latter, and vice versa for the other membrane. By inserting these membranes in the chamber as in the preceding example, they will partially separate 


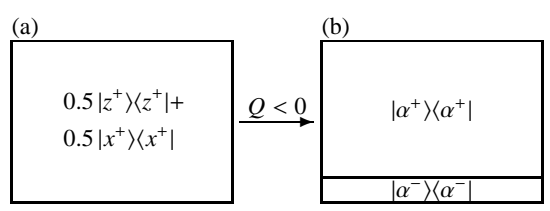

Figure 2: Separation of partially distinguishable quantum gases

and transform our $\boldsymbol{z}^{+}$- and $\boldsymbol{x}^{+}$-gases leaving in the end an $\boldsymbol{\alpha}^{+}$gas above and an $\boldsymbol{\alpha}^{-}$-gas below the two (adjoined) membranes (Fig. 2). The gases will have same the pressure but occupy unequal volumes because the ratio for both $z^{+}$- and $\boldsymbol{x}^{+}$-gases to be transformed into an $\boldsymbol{\alpha}^{+}$-gas and an $\boldsymbol{\alpha}^{-}$-gas is approximately $0.854 / 0.146$, as seen from Eqs. (13); this will also be the ratio of the final volumes. The total amount of work necessary to perform this separation is

$$
\begin{array}{r}
-0.146 n R T \ln \frac{0.146 V}{V}-0.854 n R T \ln \frac{0.854 V}{V} \approx \\
0.416 n R T,
\end{array}
$$

where the first term is for the upper membrane (transparent to the $\alpha^{+}$-gas) and the second for the lower one (transparent to the $\alpha^{-}$-gas). This is also the amount of heat released by the gases, and we see that it is less than the amount for the previous case, Eq. (7). Of course, in the reverse process, in which we mix two initially separated $\boldsymbol{\alpha}^{ \pm}$-gases in the same amounts, the gases would absorb the same (positive) amount of heat.

We shall in a moment examine how Peres uses such semipermeable membranes to show that if we could distinguish and separate two quantum-ideal-gas samples characterised by non-orthogonal statistical matrices, then a violation of the second law would follow. Although he explicitly adopts von Neumann's entropy formula, his demonstration really only uses the assumption that the thermodynamic entropy depends on the values of the parameters describing the phenomena in question at a particular instant but not on their history (as instead is the case in more general thermodynamic and thermomechanic processes [31]), so that in a cyclic process, in which we start from and end in a situation described by identical parameter values, the entropy change is naught:

$$
\Delta S=0 \quad \text { (cyclic process) }
$$

We shall also make this same sole assumption, and since it does not require a specific formula for the entropy (such as von Neumann's formula), we shall not make use of any particular entropy expression.

Finally, let us recall that the second law of thermodynamics for isothermal processes says that the amount of heat $Q a b$ sorbed by a thermodynamic body, divided by the temperature
$T$, is bounded above by the change in entropy: ${ }^{12}$

$$
Q / T \leqslant \Delta S \quad \text { (isothermal process). }
$$

This assumes in our case for a cyclic process the form ${ }^{13}$

$$
Q / T \leqslant 0=\Delta S \quad \text { (isothermal cyclic process). }
$$

\section{PERES' DEMONSTRATION}

Peres' demonstration [2, pp. 275-277] can be presented as follows. The physicist Tatiana describes the internal quantum degrees of freedom of her ideal gases by means of a spin-1/2 statistical-matrix space (with the related set of POVMs). She starts (Fig. 3, step a) with two quantum ideal gases equally divided into two compartments having volumes $V / 2$ each and separated by an impermeable membrane. In the upper compartment there is a $z^{+}$-gas, in the lower a $\boldsymbol{x}^{+}$-gas, where $z^{+}$ and $\boldsymbol{x}^{+}$are the statistical matrices defined in the previous section, Eqs. (4) and (8). Since they are non-orthogonal, $\operatorname{tr}\left(z^{+} x^{+}\right) \neq 0$, there are no means to distinguish with certainty the two gases. This implies for Tatiana the non-existence of two semi-permeable membranes with the property of being, the one, completely transparent to the $z^{+}$-gas and completely opaque to the $\boldsymbol{x}^{+}$-gas, and vice versa for the other, as discussed in the previous section.

Enters a "wily inventor", as Peres calls him [2, p. 275]; let us call him Willard. He claims having produced two such semi-permeable membranes, which can completely distinguish and separate the two gases. By means of them he reversibly mixes the two gases, obtaining work equal to $Q^{\prime}=n R T \ln 2 \approx 0.693 n R T$, cf. Eq. (7). We have now a single chamber of volume $V$ filled with a half-half mixture of $z^{+}$and $\boldsymbol{x}^{+}$-gases (step b).

From Tatiana's point of view, the situation is now the same as that discussed in the last example of the previous section: the gas mixture is equivalent (step d) to a mixture of approximately 0.854 parts of an $\boldsymbol{\alpha}^{+}$-gas and 0.146 parts of an $\boldsymbol{\alpha}^{-}$gas, where $\boldsymbol{\alpha}^{+}$and $\boldsymbol{\alpha}^{-}$are the statistical matrices defined in Eq. (11). Tatiana uses two semi-permeable membranes to separate the two $\boldsymbol{\alpha}^{ \pm}$-gases, the $\boldsymbol{\alpha}^{+}$-gas into a 0.854 fraction of the volume $V$, and the $\alpha^{-}$-gas into the remaining 0.146 fraction (so that they have the same pressure), and spends work equal to $-Q^{\prime \prime}=-n R T(0.854 \ln 0.854+0.146 \ln 0.146) \approx$ $0.416 n R T$, cf. Eq. (14).

Tatiana then performs two operations corresponding to unitary rotations of the statistical matrices associated to the two

\footnotetext{
12 This is a special case of the Clausius-Duhem inequality [32, §258][31, lecture 2][45], which is more generally valid for inhomogeneous, nonuniform bodies and for irreversible, non necessarily isothermal processes: $\int_{B} r / T \mathrm{~d} m+\int_{\partial B} q / T \mathrm{~d} A \leqslant \int_{B} \dot{s} \mathrm{~d} m$, where $r, q, s$, are respectively the massic heating supply, the heating influx, the massic entropy of the body $B$ having surface $\partial B$, and the dot represents the substantial derivative.

${ }^{13}$ See also Serrin's [46] nice analysis of the second law for cyclic processes.
} 
gases to a common one, say $z^{+}$, so that the two compartments now contain for her the same $z^{+}$-gas; she then eliminates the membranesand reinserts another impermeable one to divide the gas into two compartments of equal volume (step e), and finally performs again an operation represented by a rotation $z^{+} \mapsto \boldsymbol{x}^{+}$of the statistical matrix associated to the gas in the lower chamber. In this way she has apparently re-established the original condition of the gases (step a), which have thus undergone a cycle. These last operations are assumed to be performable without expenditure or gain of work, hence without heat exchange either. ${ }^{14}$

Tatiana summarises the results as follows: the total entropy change is naught because the initial and final conditions are the same: $\Delta S=0$. The total heat absorbed by the gases equals the work done by them and amounts to

$$
Q=Q^{\prime}+Q^{\prime \prime} \approx(0.693-0.416) n R T=0.277 n R T>0
$$

Hence, we have a violation of the second law (17) because for Tatiana's gases

$$
Q / T>0=\Delta S
$$

Tatiana accuses Willard of having violated the second law by means of his strange semi-permeable membranes that "separate non-orthogonal states".

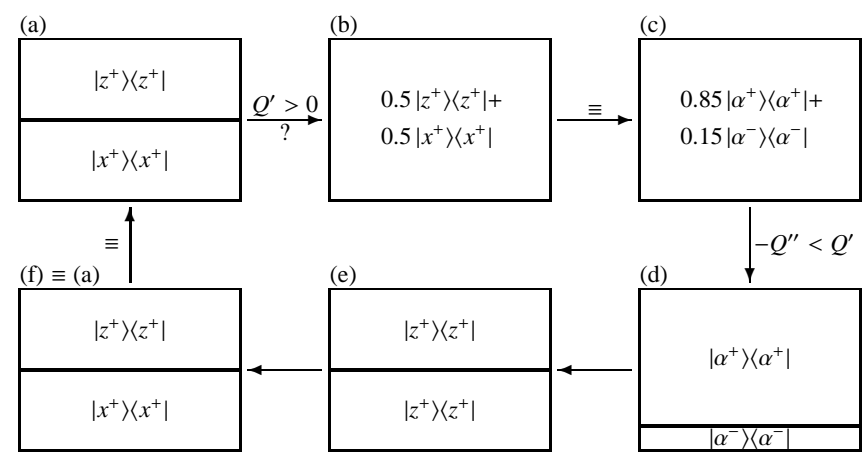

Figure 3: Quantum gas experiment from Tatiana's point of view

\footnotetext{
${ }^{14}$ Note that von Neumann [1, pp. 194 and 197] and Peres [2, p. 275] assert that unitary rotations can be realised by processes involving no heat exchange, but work exchange is allowed and indeed sometimes necessary. However, in our present discussion we have assumed all processes to be isothermal and all gases ideal, and this implies that any isochoric exchange of work must be accompanied by an equivalent exchange of heat (see $\S 3$ ); for this reason must Tatiana's final isochoric unitary rotations be performed with no energy exchange. This issue is related to the problematic way in which the quantum and classical or thermodynamical descriptions are combined; namely, the statistical matrices are not thermodynamic variables au pair with the real numbers ( $V$ and $T$ ) describing the gas.
}

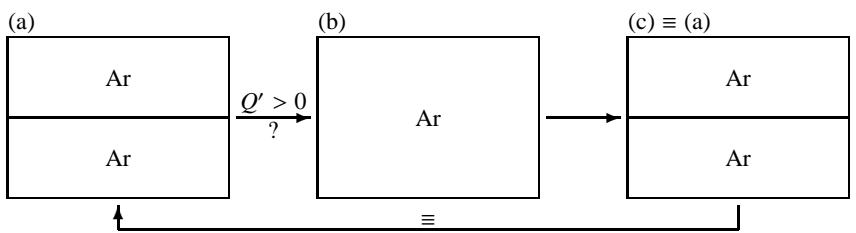

Figure 4: Classical gas experiment from Johann's point of view

\section{JAYNES' DEMONSTRATION}

We leave the quantum laboratory where Tatiana and Willard are now arguing after their experiment, and enter an adjacent classical laboratory, where we shall look at Jaynes' demonstration [14]. The situation here is in many respects very similar to the previous, though it is completely "classical".

We have an ideal gas equally divided into two chambers of volumes $V / 2$ each and separated by an impermeable membrane (Fig. 4, step a). For the scientist Johann the gas in the two chambers is exactly the same, say "ideal argon": 15 for him it would thus be impossible, not to say meaningless, to find a semi-permeable membrane that be transparent to the gas in the upper chamber and opaque to the gas in the lower one, and another membrane with the opposite properties.

The scientist Marie states nevertheless that she has in fact two membranes with those very properties. She uses them to reversibly and isothermally mix the two halves of the gas, obtaining work equal to $Q^{\prime}=n R T \ln 2 \approx 0.693 n R T$ (step b).

Yet, from Johann's point of view Marie has left things exactly how they were: he just needs to reinsert the impermeable membrane in the middle of the vessel and for him the situation is exactly the same as in the beginning: the gas is equally divided into two chambers (steps c, a).

Johann's conclusion is the following: The initial and final conditions of the gas are the same and so the total entropy change vanishes: $\Delta S=0$. The work obtained equals the heat absorbed by the gas,

$$
Q=Q^{\prime} \approx 0.693 n R T>0
$$

The second law of thermodynamics states that this amount of heat divided by the temperature cannot exceed the entropy change, ${ }^{16} Q / T \leqslant \Delta S$, but this is quite incompatible with Johann's conclusion that

$$
Q / T>0=\Delta S
$$

(cf. Tatiana's Eq. (19)).

\footnotetext{
15 Real argon, of course, behaves like an ideal gas only in certain ranges of temperature and volume.

16 Note that, strictly speaking, ideal gases (or mixtures thereof) cannot undergo irreversible processes (the usual example of "free expansion" evidently assumes that the gas is not ideal during the expansion).
} 


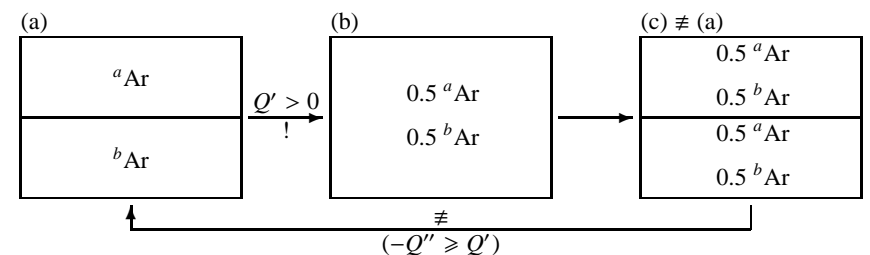

Figure 5: Classical gas experiment from Marie's point of view

Johann, however, is never dogmatic about his own knowledge of the experimental facts. Asking Marie whether she is able to reproduce her "trick" at will or whether it was only chance, and upon her answer that the separation is reproducible, he understands that where for him there was only one gas there must actually be two different gases. This is indeed the case: Marie explains that the two chambers initially contained two different kinds of ideal-argon, of which Johann had no knowledge: $\operatorname{argon}$ ' $a$ ' ( $\left.{ }^{a} \mathrm{Ar}\right)$ and argon ' $b$ ' $\left({ }^{b} \mathrm{Ar}\right)$. Argon $a$ is soluble in whafnium while $\operatorname{argon} b$ is not, but the latter is soluble in whifnium, a property not shared by the $a$ variety. ${ }^{17}$ Marie's separation of the two gases ${ }^{a} \mathrm{Ar}$ and ${ }^{b} \mathrm{Ar}$ was possible by means of two semi-permeable membranes made of whifnium and whafnium that take advantage of these different properties.

We see (Marie's point of view, Fig. 5) that the second law is not violated. Initially the two gases ${ }^{a} \mathrm{Ar}$ and ${ }^{b} \mathrm{Ar}$ were completely separated in the vessel's two chambers (step a). After mixing and extracting work, the vessel contained an equal mixture of ${ }^{a} \mathrm{Ar}$ and ${ }^{b} \mathrm{Ar}$ (step b). Upon Johann's reinsertion of the impermeable membrane the vessel is again divided in two equal chambers, but each chamber contains a mixture of ${ }^{a} \mathrm{Ar}$ and ${ }^{b} \mathrm{Ar}$ (step c), and this is different from the initial condition (step a): the cycle has not been completed although it appeared so to Johann, and so the equation $\Delta S=0$ is not necessarily valid. To close the cycle one has to use the semi-permeable membranes again to relegate the two gases to two separate chambers, and must thereby spend an amount of work $-Q^{\prime \prime}$ at least equal to that previously obtained, $-Q^{\prime \prime} \geqslant Q^{\prime}$, and the second law (16) for the completed cycle is satisfied: $Q=Q^{\prime}+Q^{\prime \prime} \leqslant 0=\Delta S$.

The simple conclusion, drawn by Jaynes [14, §3] in terms of entropy, is that

it is necessary to decide at the outset of a problem which macroscopic variables or degrees of freedom we shall measure and/or control; and within the context of the thermodynamic system thus defined, entropy will be some function $S\left(X_{1}, \ldots, X_{n}\right)$ of whatever variables we have chosen. We can expect this to obey the second

\footnotetext{
17 Jaynes explains that 'whifnium', as well as 'whafnium', 'is one of the rare superkalic elements; in fact, it is so rare that it has not yet been discovered" $[14, \S 5]$.
}

law $[Q / T \leqslant \Delta S]$ only as long as all experimental manipulations are confined to that chosen set. If someone, unknown to us, were to vary a macrovariable $X_{n+1}$ outside that set, he could produce what would appear to us as a violation of the second law, since our entropy function $S\left(X_{1}, \ldots, X_{n}\right)$ might decrease spontaneously, while his $S\left(X_{1}, \ldots, X_{n}, X_{n+1}\right)$ increases.

This is old wisdom; for example, Grad had explained thirtyone years earlier that $[25$, p. 325] (see also [24, 26])

the adoption of a new entropy is forced by the discovery of new information. [...] The existence of diffusion between oxygen and nitrogen somewhere in a wind tunnel will usually be of no interest. Therefore the aerodynamicist uses an entropy which does not recognise the separate existence of the two elements but only that of "air". In other circumstances, the possibility of diffusion between elements with a much smaller mass ratio (e.g., 238/235) may be considered quite relevant.

We can rephrase Grad's and Jaynes' remark shifting the emphasis to the distinction between the experimental situation and the mathematics which describes it: The fact that some physicist can perform experimental operations which contradict our mathematical description and which apparently lead to violations of e.g. the second law, simply means that that physicist is able to control physical phenomena which are not contemplated by our mathematical description, and the second law is not necessarily violated in that physicist's more appropriate mathematical description.

\section{RE-ANALYSIS OF PERES' DEMONSTRATION}

With the insight provided by Grad and Jaynes, we can return to the quantum laboratory and look with different eyes at what happened there. If Willard can reproducibly distinguish and separate with certainty the two physical preparations represented by Tatiana through the non-orthogonal statistical matrices $\boldsymbol{z}^{+}$and $\boldsymbol{x}^{+}$, this can only have one meaning: these preparations have to be represented by orthogonal statistical matrices instead, at least in experimental situations in which Willard takes advantage of his instrumental capabilities, his "tricks". This is not in contradiction with Tatiana's formalism: with the instruments and apparatus at her disposal the two physical situations are not distinguishable with certainty, and so the appropriate way for her of representing them was by non-orthogonal statistical matrices. But she can now share Willard's instrumentation and knowledge and use an accordingly more adequate mathematical description of the physical facts.

We can imagine a possible explanation from Willard's point of view (it is just a possible one, and even more drastic ones, 
requiring abandonment of the quantum-mechanical formalism, might be necessary in other instances). Willard explains that the internal quantum degrees of freedom of the gases are best represented by a spin-3/2 statistical-matrix space, of which Tatiana used a subspace (more exactly, a projection) because of her limited observational means; i.e., part of the statistical-matrix space was "traced out" because Tatiana used only instrumentation represented by a portion of the total POVM space. For instance, denoting by $\left\{\left|\tilde{z}^{+}\right\rangle,\left|\tilde{z}^{-}\right\rangle,\left|z^{+}\right\rangle,\left|z^{-}\right\rangle\right\}$ the basis for the Hilbert space used by Willard, with $\left\langle\tilde{z}^{+}\right.$| $\left.z^{+}\right\rangle=\left\langle\tilde{z}^{-} \mid z^{-}\right\rangle \equiv 0$, Tatiana could not distinguish, amongst others, the preparations corresponding to $\left|\tilde{z}^{+}\right\rangle$and to $\left|z^{+}\right\rangle$, both of which she represented as $\left|z^{+}\right\rangle$, nor those corresponding to $\left|\tilde{x}^{+}\right\rangle \stackrel{\text { def }}{=}\left(\left|\tilde{z}^{+}\right\rangle+\left|\tilde{z}^{-}\right\rangle\right) / \sqrt{2}$ and to $\left|\grave{x}^{+}\right\rangle \stackrel{\text { def }}{=}\left(\left|z^{+}\right\rangle+\left|\grave{z}^{-}\right\rangle\right) / \sqrt{2}$, which she denoted as $\left|x^{+}\right\rangle$. The projection is thus

$$
\left|\tilde{z}^{+}\right\rangle \mapsto\left|z^{+}\right\rangle, \quad\left|\tilde{z}^{-}\right\rangle \mapsto\left|z^{-}\right\rangle, \quad\left|\dot{z}^{+}\right\rangle \mapsto\left|z^{+}\right\rangle, \quad\left|\dot{z}^{-}\right\rangle \mapsto\left|z^{-}\right\rangle,
$$

from which also follows

$$
\left|\tilde{x}^{+}\right\rangle \mapsto\left|x^{+}\right\rangle, \quad\left|\tilde{x}^{-}\right\rangle \mapsto\left|x^{-}\right\rangle, \quad\left|\hat{x}^{+}\right\rangle \mapsto\left|x^{+}\right\rangle, \quad\left|\hat{x}^{-}\right\rangle \mapsto\left|x^{-}\right\rangle,
$$

which makes it evident that Tatiana cannot distinguish preparations and experiments represented by the vectors with a tilde from the corresponding ones represented by accented vectors. $^{18}$

Thus, the preparations which Tatiana represents by $z^{+}$and $\boldsymbol{x}^{+}$because for her they were indistinguishable, are instead represented by Willard by

$$
\tilde{z}^{+} \stackrel{\text { def }}{=}\left|\tilde{z}^{+}\right\rangle\left\langle\tilde{z}^{+}\left|\hat{=}\left(\begin{array}{llll}
1 & 0 & 0 & 0 \\
0 & 0 & 0 & 0 \\
0 & 0 & 0 & 0 \\
0 & 0 & 0 & 0
\end{array}\right), \quad \grave{x}^{+} \stackrel{\text { def }}{=}\right| \grave{x}^{+}\right\rangle\left\langle\grave{x}^{+}\right| \hat{=}\left(\begin{array}{llll}
0 & 0 & 0 & 0 \\
0 & 0 & 0 & 0 \\
0 & 0 & 1 & 0 \\
0 & 0 & 0 & 0
\end{array}\right),
$$

which are clearly orthogonal, $\operatorname{tr}\left(\tilde{z}^{+} \grave{\boldsymbol{x}}^{+}\right)=0$, because for him the two corresponding preparations are distinguishable. From his point of view, the process went as follows. The two compartments initially contained $\tilde{z}^{+}$- and $\grave{x}^{+}$-gases (Fig. 6, step a). With his semi-permeable membranes he mixed the two distinguishable gases with extraction of work (step b), so that the chamber eventually contained a $\tau$-gas with

$$
\tau=\frac{1}{2} \tilde{z}^{+}+\frac{1}{2} \grave{x}^{+} \hat{=} \frac{1}{2}\left(\begin{array}{llll}
1 & 0 & 0 & 0 \\
0 & 0 & 0 & 0 \\
0 & 0 & 1 & 0 \\
0 & 0 & 0 & 0
\end{array}\right) \text {. }
$$

Tatiana's subsequent separation by means of her semipermeable membranes (steps c, d), distinguishing the preparations corresponding to $\boldsymbol{\alpha}^{+}$and $\boldsymbol{\alpha}^{-}$, is represented by Willard by the POVM

$$
\begin{aligned}
\boldsymbol{E}^{ \pm} & \stackrel{\text { def }}{=}\left|\tilde{\alpha}^{ \pm}\right\rangle\left\langle\tilde{\alpha}^{ \pm}|+| \grave{\alpha}^{ \pm}\right\rangle\left\langle\grave{\alpha}^{ \pm}\right|, \\
& \hat{=} \frac{1}{4}\left(\begin{array}{cccc}
2 \pm \sqrt{2} & \pm \sqrt{2} & 0 & 0 \\
\pm \sqrt{2} & 2 \mp \sqrt{2} & 0 & 0 \\
0 & 0 & 2 \pm \sqrt{2} & \pm \sqrt{2} \\
0 & 0 & \pm \sqrt{2} & 2 \mp \sqrt{2}
\end{array}\right),
\end{aligned}
$$

\footnotetext{
${ }^{18}$ An even more evident notation would be $\left|\tilde{z}^{+}\right\rangle \equiv\left|z^{+}, z^{+}\right\rangle,\left|z^{+}\right\rangle \equiv\left|z^{+}, z^{-}\right\rangle$,
} etc., but it might be misleading in other respects. with

$$
\begin{aligned}
& \left|\tilde{\boldsymbol{\alpha}}^{ \pm}\right\rangle \stackrel{\text { def }}{=}(2 \pm \sqrt{2})^{-\frac{1}{2}}\left(\left|\tilde{z}^{ \pm}\right\rangle \pm\left|\tilde{x}^{ \pm}\right\rangle\right), \\
& \left|\grave{\boldsymbol{\alpha}}^{ \pm}\right\rangle \stackrel{\text { def }}{=}(2 \pm \sqrt{2})^{-\frac{1}{2}}\left(\left|\dot{z}^{ \pm}\right\rangle \pm\left|\grave{x}^{ \pm}\right\rangle\right)
\end{aligned}
$$

cf. Eq. (11), and the associated CPMs $\tau \mapsto$ $\boldsymbol{E}^{ \pm} \boldsymbol{\tau} \boldsymbol{E}^{ \pm} / \operatorname{tr}\left(\boldsymbol{E}^{ \pm} \boldsymbol{\tau} \boldsymbol{E}^{ \pm}\right)$.

That separation led to a compartment, with volume $0.854 \mathrm{~V}$, containing the gas mixture

$$
\frac{1}{2}\left|\tilde{\alpha}^{+}\right\rangle\left\langle\tilde{\alpha}^{+}\left|+\frac{1}{2}\right| \grave{\alpha}^{+}\right\rangle\left\langle\grave{\alpha}^{+}\right|,
$$

and the other compartment, with volume $0.146 \mathrm{~V}$, containing the gas mixture

$$
\frac{1}{2}\left|\tilde{\alpha}^{-}\right\rangle\left\langle\tilde{\alpha}^{-}\left|+\frac{1}{2}\right| \grave{\alpha}^{-}\right\rangle\left\langle\grave{\alpha}^{-}\right| .
$$

Tatiana could not perceive that these were mixtures, because of her limited instrumentation.

The following step corresponded to the rotations

$$
\left|\tilde{\alpha}^{+}\right\rangle\left\langle\tilde{\alpha}^{+}|\mapsto| \tilde{z}^{+}\right\rangle\left\langle\tilde{z}^{+}|, \quad| \grave{\alpha}^{+}\right\rangle\left\langle\grave{\alpha}^{+}|\mapsto| \grave{z}^{+}\right\rangle\left\langle\grave{z}^{+}\right|
$$

for gases in the upper compartment, and

$$
\left|\tilde{\alpha}^{-}\right\rangle\left\langle\tilde{\alpha}^{-}|\mapsto| \tilde{z}^{+}\right\rangle\left\langle\tilde{z}^{+}|, \quad| \grave{\alpha}^{-}\right\rangle\left\langle\grave{\alpha}^{-}|\mapsto| \grave{z}^{+}\right\rangle\left\langle\grave{z}^{+}\right|
$$

for the gases in the lower compartment (remember that $\left\langle\tilde{z}^{+} \mid z^{+}\right\rangle=0$ ). The successive elimination and reinsertion of the impermeable membrane led to two compartments of equal volumes $V / 2$ and equal content, viz. the equal mixture of $\left|\tilde{z}^{+}\right\rangle\left\langle\tilde{z}^{+}\right|$- and $\left|z^{+}\right\rangle\left\langle\bar{z}^{+}\right|$-gases (step e).

The final rotation for the gas in the lower compartment,

$$
\left|\tilde{z}^{+}\right\rangle \mapsto\left|\tilde{x}^{+}\right\rangle, \quad\left|\grave{z}^{+}\right\rangle \mapsto\left|\grave{x}^{+}\right\rangle
$$

(remember that $\left\langle\tilde{x}^{+} \mid \grave{x}^{+}\right\rangle=0$ ), only led to two equal compartments containing the mixtures of $\frac{1}{2}\left|\tilde{z}^{+}\right\rangle\left\langle\tilde{z}^{+}\left|+\frac{1}{2}\right| z^{+}\right\rangle\left\langle\bar{z}^{+}\right|$and

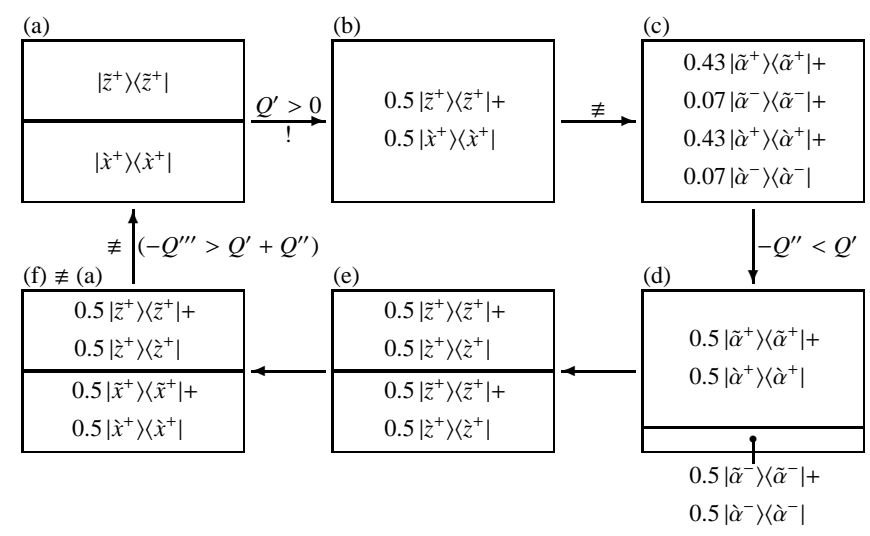

Figure 6: Quantum gas experiment from Willard's point of view 
$\frac{1}{2}\left|\tilde{x}^{+}\right\rangle\left\langle\tilde{x}^{+}\left|+\frac{1}{2}\right| \grave{x}^{+}\right\rangle\left\langle\grave{x}^{+}\right|$gases respectively (step f). This is of course different from the initial situation (step a); but for Tatiana, whose instrumentation was limited with respect to Willard's, the initial and final conditions appeared identical.

The second law is not violated, because the cycle has not been completed, and the equation $\Delta S=0$ does thus not necessarily hold. It is easy to see that in order to return to the initial condition an amount of work $-Q^{\prime \prime \prime} \geqslant 4 \times(1 / 4) n R T \ln 2 \approx$ $0.693 n R T$ has to be spent to separate the $\left|\tilde{z}^{+}\right\rangle\left\langle\tilde{z}^{+}\right|$-gas from the $\left|\grave{z}^{+}\right\rangle\left\langle\grave{z}^{+}\right|$-gas, and analogously for the $\left|\tilde{x}^{+}\right\rangle\left\langle\tilde{x}^{+}\right|-$and $\left|\grave{x}^{+}\right\rangle\left\langle\grave{x}^{+}\right|-$ gases. A final operation must then be performed corresponding to the rotations of the statistical matrices $\left|z^{+}\right\rangle\left\langle z^{+}\right|$and $\left|\tilde{x}^{+}\right\rangle\left\langle\tilde{x}^{+}\right|$to $\left|\tilde{z}^{+}\right\rangle\left\langle\tilde{z}^{+}\right|$and $\left|\hat{x}^{+}\right\rangle\left\langle\hat{x}^{+}\right|$respectively, and we have finally reached again the initial condition (step a). The total amount of heat absorbed by the gases, corresponding to the work performed on them would then be

$$
\begin{array}{r}
Q=Q^{\prime}+Q^{\prime \prime}+Q^{\prime \prime \prime} \leqslant(0.693-0.416-0.693) n R T= \\
-0.416 n R T \leqslant 0=\Delta S,
\end{array}
$$

and the second law, for the completed cycle, is satisfied (strictly so: we see that the whole process is irreversible, and it is easy to check that the only irreversible step was the separation performed by Tatiana into $\boldsymbol{\alpha}^{+}$- and $\boldsymbol{\alpha}^{-}$-gases).

\section{CONCLUSION}

The re-analysis, with Jaynes' (and Grad's) insight, of the simple quantum experiment which seemed to violate the second law leads to the following almost trivial conclusion: if the physicist Willard can reproducibly distinguish two physical preparations that the physicist Tatiana represents by nonorthogonal statistical matrices, then no necessary violation of the second law of thermodynamics is implied from Willard's point of view. On the other hand it is certain that the particular statistical-matrix space adopted by Tatiana for the phenomenon's description is not (any longer) adequate, and has to be amended (in extreme cases a non-quantum-mechanical description might be necessary) in order to avoid inconsistencies like e.g. seeming violations of the second law. Alternatively, Tatiana can keep on using the unamended mathematical description, but she must then renounce to treat with it situations involving the new experimental possibility and the related phenomena, in order to avoid inconsistencies.

This conclusion emphasises the distinction, somewhat obscured in von Neumann's statements but often stressed by Peres [2, 11, 43, 47] and Jaynes [23] amongst others, ${ }^{19}$ between physical phenomena and their mathematical description: "quantum phenomena do not occur in a Hilbert space.

\footnotetext{
${ }^{19}$ E.g. Ekstein [3, 4], Giles [5-7], Foulis and Randall [8-10], Band and Park [12, 13]; cf. also References [15-17].
}

They occur in a laboratory" [47]. ${ }^{20}$ (The present author is in fact guilty of unclarity about this important distinction in a previous paper [48].) The usual metonymic expression ${ }^{21}$ "to distinguish two statistical matrices" is certainly handy, but must be used with a grain of salt: what we distinguish is in fact two physical situations, facts, phenomena, or preparations; not two statistical matrices. The latter should mathematically reflect what we can do with these preparations, e.g.whether we can distinguish them, and be amended whenever new experimental facts appear. ${ }^{22}$

\section{Acknowledgements}

I thank Gunnar Björk and Anders Månsson for many discussions regarding von Neumann's demonstration, Gunnar also for encouragement and for precious comments on the paper, Louise and Anna for encouragement, and Louise for invaluable odradek.

[1] J. von Neumann, Mathematische Grundlagen der Quantenmechanik (Springer-Verlag, Berlin, 1996), 2nd ed., first publ. 1932, transl. by Robert T. Beyer as Mathematical Foundations of Quantum Mechanics (Princeton University Press, Princeton, 1955).

[2] A. Peres, Quantum Theory: Concepts and Methods (Kluwer Academic Publishers, Dordrecht, 1995).

[3] H. Ekstein, Presymmetry, Phys. Rev. 153, 1397-1402 (1967).

[4] H. Ekstein, Presymmetry. II, Phys. Rev. 184, 1315-1337 (1969).

[5] R. Giles, Foundations for quantum statistics, J. Math. Phys. 9, 357-371 (1968).

[6] R. Giles, Foundations for quantum mechanics, J. Math. Phys. 11, 2139-2160 (1970).

[7] R. Giles, Formal languages and the foundations of physics, in C. A. Hooker, ed., Physical Theory as Logico-Operational Structure (D. Reidel Publishing Company, Dordrecht, 1979), pp. 19-87.

[8] D. J. Foulis and C. H. Randall, Operational statistics. I. Basic concepts, J. Math. Phys. 13, 1667-1675 (1972).

[9] C. H. Randall and D. J. Foulis, Operational statistics. II. Manuals of operations and their logics, J. Math. Phys. 14, 1472-1480 (1973).

${ }^{20}$ A similar explicit observation had already been made by Band and Park [12]: "experimenters do not apprehend Hilbert vectors; they gather numerical data" (their emphasis).

${ }^{21}$ Metonymy is "a figure of speech consisting of the use of the name of one thing for that of another of which it is an attribute or with which it is associated (as 'crown' in 'lands belonging to the crown')" (Merriam-Webster Online Dictionary); it perfectly applies to our case.

${ }^{22}$ We must not forget, however, that the mathematical formalism is often expanded with no or very little experimental input and successfully leads thus to new discoveries (think of e.g. general relativity, Dirac's positron, or the Clausius-Duhem inequality). 
[10] D. J. Foulis and C. H. Randall, Manuals, morphisms and quantum mechanics, in A. R. Marlow, ed., Mathematical Foundations of Quantum Theory (Academic Press, London, 1978), pp. $105-126$.

[11] A. Peres, What is a state vector?, Am. J. Phys. 52, 644-650 (1984).

[12] J. L. Park and W. Band, Mutually exclusive and exhaustive quantum states, Found. Phys. 6, 157-172 (1976).

[13] W. Band and J. L. Park, New information-theoretic foundations for quantum statistics, Found. Phys. 6, 249-262 (1976).

[14] E. T. Jaynes, The Gibbs paradox, in Maximum-Entropy and Bayesian Methods, edited by C. R. Smith, G. J. Erickson, and P. O. Neudorfer (Kluwer Academic Publishers, Dordrecht, 1992), pp. 1-22, http://bayes.wustl.edu/etj/ articles/gibbs. paradox.pdf.

[15] L. E. Ballentine, The statistical interpretation of quantum mechanics, Rev. Mod. Phys. 42, 358 (1970).

[16] L. Hardy, Quantum theory from five reasonable axioms (2001), arxiv.org/quant-ph/0101012.

[17] P. G. L. Mana, Probability tables, in A. Y. Khrennikov, ed., Quantum Theory: Reconsideration of Foundations - 2 (Växjö University Press, Växjö, Sweden, 2004), pp. 387-401, see also arxiv.org/quant-ph/0403084.

[18] E. P. Wigner, On the quantum correction for thermodynamic equilibrium, Phys. Rev. 40, 749-759 (1932).

[19] U. Fano, Description of states in quantum mechanics by density matrix and operator techniques, Rev. Mod. Phys. 29, 74-93 (1957).

[20] I. M. Copi and C. Cohen, Introduction to Logic (MacMillan Publishing Company, New York, 1990), eighth ed., first publ. 1953.

I. M. Copi, Symbolic Logic (Macmillan Publishing, New York, 1979), 5th ed., first publ. 1954.

[21] E. T. Jaynes, Clearing up mysteries - the original goal, in Maximum Entropy and Bayesian Methods, edited by J. Skilling (Kluwer Academic Publishers, Dordrecht, 1989), pp. 1-27, http://bayes. wustl . edu/etj/node1.html.

[22] E. T. Jaynes, Probability theory as logic, in Proceedings of the Ninth Annual Workshop on Maximum Entropy and Bayesian Methods, edited by P. Fougère (Kluwer Academic Publishers, Dordrecht, 1990), revised, corrected, and extended version at http://bayes. wustl. edu/etj/node1.html.

[23] E. T. Jaynes, Probability Theory: The Logic of Science (Cambridge University Press, Cambridge, 2003), ed. by G. Larry Bretthorst, first publ. 1995 at http://bayes.wustl. edu/etj (chapter 30, Maximum entropy: matrix formulation, of the original version of the book, not included in the printed one, is available at http://www.imit.kth.se/ mana/work/cc30e.pdf).

[24] H. Grad, Statistical mechanics of dynamical systems with integrals other than energy, J. Phys. Chem. 56, 1039-1048 (1952).

[25] H. Grad, The many faces of entropy, Comm. Pure Appl. Math. 14, 323-354 (1961).

[26] H. Grad, Levels of description in statistical mechanics and thermodynamics, in M. Bunge, ed., Delaware Seminar in the Foundations of Physics (Springer-Verlag, Berlin, 1967), pp. 49-76.

[27] C. A. Truesdell, III and S. Bharatha, The Concepts and Logic of Classical Thermodynamics as a Theory of Heat Engines: Rigorously Constructed upon the Foundation Laid by S. Carnot and F. Reech (Springer-Verlag, New York, 1977).

[28] J. R. Partington, An Advanced Treatise on Physical Chemistry. Vol. I: Fundamental Principles. The Properties of Gases (Long- mans, Green and Co, London, 1949).

[29] J. W. Gibbs, On the equilibrium of heterogeneous substances, Trans. Connect. Acad. 3, 108-248, 343-524 (1876-1878), repr. in The Collected Works of J. Willard Gibbs. Vol. 1: Thermodynamics (Yale University Press, New Haven, 1948, first publ. 1906), pp. 55-353.

[30] H. A. Buchdahl, The Concepts of Classical Thermodynamics (Cambridge University Press, London, 1966).

[31] C. A. Truesdell, III, Rational Thermodynamics (SpringerVerlag, New York, 1984), 2nd ed., first publ. 1969.

[32] C. A. Truesdell, III and R. A. Toupin, The classical field theories, in S. Flügge, ed., Handbuch der Physik: Band III/1: Prinzipien der klassischen Mechanik und Feldtheorie [Encyclopedia of Physics: Vol. III/1: Principles of Classical Mechanics and Field Theory] (Springer-Verlag, Berlin, 1960), pp. 226858.

[33] R. M. Bowen, Thermochemistry of reacting materials, J. Chem. Phys. 49, 1625-1637 (1968).

[34] C. A. Truesdell, III, Sulle basi della termodinamica delle miscele, Atti Accad. Naz. Lincei: Rend. Sc. fis. mat. nat. 44, 381383 (1968).

[35] I. Samohýl, Thermodynamics of Irreversible Processes in Fluid Mixtures (Approached by Rational Thermodynamics) (BSB B. G. Teubner Verlagsgesellschaft, Leipzig, 1987).

[36] I. Samohýl, Thermodynamics of reacting mixtures of any symmetry with heat conduction, diffusion and viscosity, Arch. Rational Mech. Anal. 147, 1-45 (1999).

[37] A. Einstein, Beiträge zur Quantentheorie, Ver. Deut. Phys. Ges. 16, 820-828 (1914), transl. by A. Engel and E. Schucking as Contributions to quantum theory, in The Collected Papers of Albert Einstein: Vol. 6: The Berlin Years: Writings, 19141917. (English translation) (Princeton University Press, Princeton, 1997), pp. 20-26.

[38] G. Björk, P. G. L. Mana, and A. Månsson (2005), in preparation.

[39] J. Larmor, A dynamical theory of the electric and luminiferous medium. - Part III. Relations with material media, Phil. Trans. R. Soc. Lond. A 190, 205-300, 493 (1897), repr. in J. Larmor, Mathematical and Physical Papers. Vol. II (Cambridge University Press, Cambridge, 1929), pp. 11-131.

[40] D. Dieks and V. van Dijk, Another look at the quantum mechanical entropy of mixing, Am. J. Phys. 56, 430-434 (1988).

[41] E. B. Davies, Quantum Theory of Open Systems (Academic Press, London, 1983).

[42] K. Kraus, States, Effects, and Operations: Fundamental Notions of Quantum Theory (Springer-Verlag, Berlin, 1983).

[43] A. Peres, Classical interventions in quantum systems. I. The measuring process, Phys. Rev. A 61, 022116 (2000), arxiv. org/quant-ph/9906023.

[44] A. Peres, Classical interventions in quantum systems. II. Relativistic invariance, Phys. Rev. A 61, 022117 (2000), arxiv. org/quant-ph/9906034.

[45] M. Šilhavý, The Mechanics and Thermodynamics of Continuous Media (Springer-Verlag, Berlin, 1997).

[46] J. Serrin, Conceptual analysis of the second law of thermodynamics, Arch. Rational Mech. Anal. 70, 355-371 (1979).

[47] A. Peres, What's wrong with these observables? (2002), arxiv.org/quant-ph/0207020.

[48] P. G. L. Mana, Why can states and measurement outcomes be represented as vectors? (2003), arxiv.org/quant-ph/ 0305117. 